\title{
Reversible Atmospheric Water Harvesting Using Metal-Organic Frameworks
}

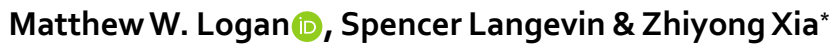

The passive capture of clean water from humid air without reliance on bulky equipment and high energy has been a substantial challenge and has attracted significant interest as a potential environmentally friendly alternative to traditional water harvesting methods. Metal-organic frameworks (MOFs) offer a high potential for this application due to their structural versatility which permits scalable, facile modulations of structural and functional elements. Although MOFs are promising materials for water harvesting, little research has been done to address the microstructure-adsorbing characteristics relationship with respect to the dynamic adsorption-desorption process. In this article, we present a parametric study of nine hydrolytically stable MOFs with diverse structures for unraveling fundamental material properties that govern the kinetics of water sequestration in this class of materials as well as investigating overall uptake capacity gravimetrically. The effects of temperature, relative humidity, and powder bed thickness on the adsorption-desorption process are explored for achieving optimal operational parameters. We found that Zr-MOF-808 can produce up to $8.66 \mathrm{~L}_{\mathrm{H} 2 \mathrm{O}} \mathrm{kg}^{-1} \mathrm{MOF}$ day ${ }^{-1}$, an extraordinary finding that outperforms any previously reported values for MOF-based systems. The presented findings help to deepen our understanding and guide the discovery of next-generation water harvesting materials.
\end{abstract}

Despite the rapid growth of modern infrastructure, access to clean water remains a critical issue and challenge to humanity that is projected to increase at a rate faster than that of energy production ${ }^{1}$. Limited access to freshwater due to the absence of sources, such as lakes, rivers, and groundwater is becoming even more problematic, with many of these sources becoming contaminated from human activities. Traditional means to acquire clean water, such as reverse osmosis and distillation, is costly and energy-intensive which in turn restricts real-world uses ${ }^{2,3}$. Therefore, it is essential to find portable solutions that are simple and low cost to produce clean water on demand in various environments.

A substantial effort on accessing nontraditional water reserves, such as atmospheric water vapor, focuses on the ability to supply freshwater on-demand virtually anywhere on the earth ${ }^{4}$. Atmospheric water harvesting offers an attractive alternative by providing access to the omnipresent water vapor in the earth's atmosphere off the grid and in virtually any environment. Direct water harvesting from air has been demonstrated through cooling water vapor below its saturation pressure is not practical in dry climates due to its high energy demands ${ }^{3}$.

Metal-organic frameworks (MOFs) due to their unique micro-structure, intrinsic porosity, and unprecedented functional and chemical control have a high potential to be used for harvesting water from air ${ }^{5,6}$. Recently, the use of MOFs to leverage the earth's natural thermal swing process to efficiently sequester clean water with little to no additional energy input has been demonstrated ${ }^{7}$. MOFs are commonly constructed through the self-assembly of metal oxide clusters (also known as nodes) and multifunctional organic linkers (also known as struts), and form highly crystalline complex 3D architectures. The modularity in MOFs allows the properties of these distinct hierarchical structures to be tunable from the molecular level of the inorganic node and/or organic linker to that of a microscopic level and subsequently particle morphology. Recently, Hanikel et al. demonstrated that a water generation capability of $1.3 \mathrm{~L} \mathrm{~kg}_{\mathrm{MOF}}^{-1}$ day $^{-1}$ was achieved by using Al-MOF-303 at 32\% relative humidity (RH) and $27^{\circ} \mathrm{C}$ using multiple adsorption-desorption sequences ${ }^{4}$. Even though the water recovery rate is low, their system is a breakthrough in the rapid production of water from the atmosphere using multiple harvesting cycles per day without the need of energy inputs. This work reveals the significance of water uptake dynamics, as opposed to the majority of previous studies that focused on controlling the adsorption equilibrium and total water capacity 


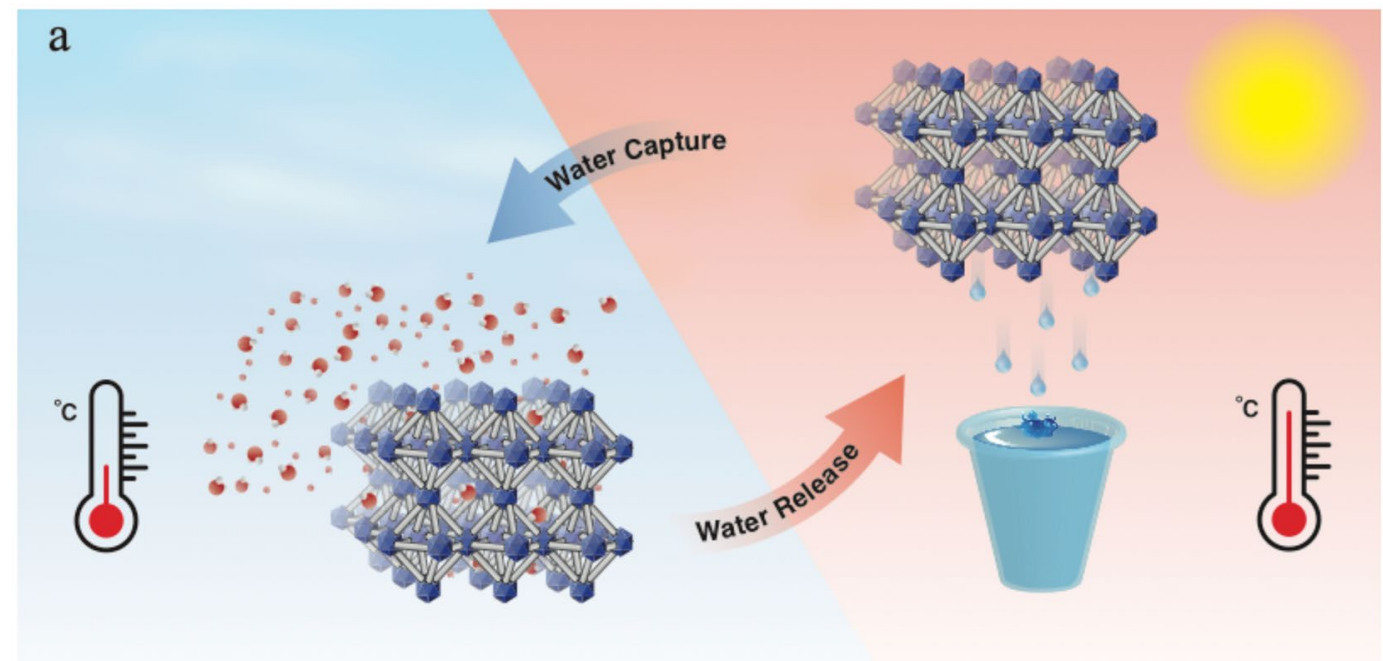

b
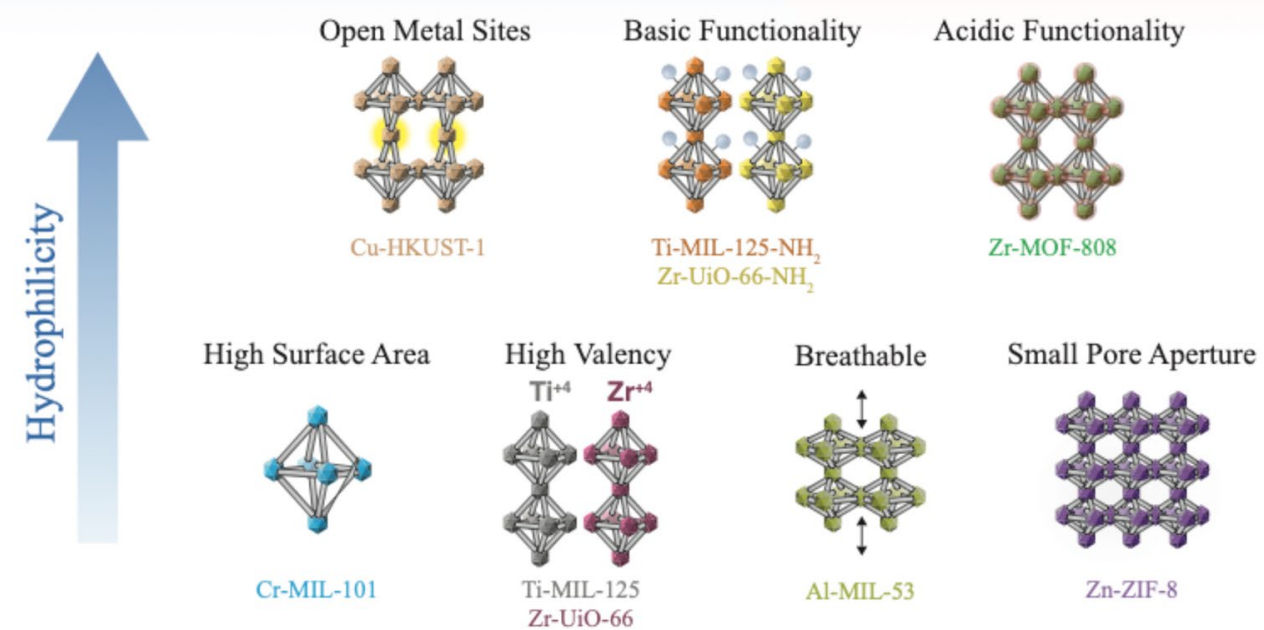

Cr-MIL-101

Figure 1. Schematic illustration of the MOF water harvesting system (a) and a general overview of MOFs employed in the present study (b), highlighting different functionalities and representative MOFs.

in materials ${ }^{8-11}$. Although these are essential characteristics for water sequestering materials to possess, the more valuable property is fast and efficient production of water. Consequently, understanding the structure-property relationship controlling these effects is vital for the creation of optimized water harvesting materials based on MOFs.

In this study, we evaluated the effects of relative humidity, temperature, and the dynamic sorption properties on a wide range of MOFs with different nodes and strut functionalities. The structure-property relationship and its role in the water capture and release kinetics are carefully addressed under several well controlled conditions. Our ultimate goal is to develop a design strategy for ambient water harvesting systems and guide the development of next-generation water harvesting materials.

\section{Results and Discussion}

The MOF-based water harvesting system is schematically shown in Fig. 1a, which has been simplified to highlight the water harvesting using MOF. Further, the capture and release cycles occur independently in regular cycles using a thermal swing process. Figure $1 \mathrm{~b}$ shows the simplified morphologies of the MOFs used in this study. The details of the MOF synthesis can be found in the methods section. In-depth characterization of all these MOFs can be found in the Supplementary Information. Briefly, Al-MIL-53 ${ }^{12}$ has a distinctive breathing capability with moderate accessible surface area; Ti-MIL-125 ${ }^{13}, \mathbf{Z r}-\mathbf{U i O}-\mathbf{6 6}^{14}$, and Cr-MIL-101 ${ }^{15}$ contain high valence metal-oxide clusters and hard carboxylate-based organic linkers owing to their excellent chemical stability and limited chemical functionality that restricts polarity in the view of this study. Ti-MIL-125- $\mathbf{N H}_{2}{ }^{16}$ and $\mathbf{Z r}-\mathrm{UiO}-$ 66- $\mathrm{NH}_{2}{ }^{14}$ are the respective isoreticular motifs to Ti-MIL-125 and $\mathrm{Zr}$-UiO-66 with polar and basic functionalities substituted on the diatopic organic linker. Zr-MOF-808 possesses an acidic pore and high surface $\operatorname{area}^{17}$. Zn-ZIF-8 contains polar properties but with narrow pore apertures $(3.4 \AA)$ that exhibit hydrophobic properties ${ }^{18}$. Cu-HKUST-1 is constructed by a tritopic organic moiety and copper paddle-wheel secondary building units (SBUs) with open metal sites ${ }^{19}$.

To simulate the real world conditions, the maximum amount of harvested water is not determined by the uptake equilibrium, but rather by the amount of the water that can be desorbed after saturation. In this work, 
a

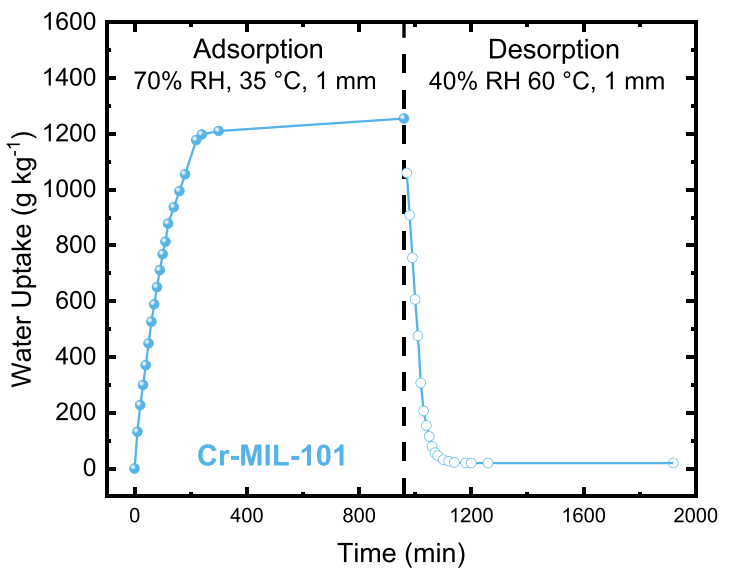

b

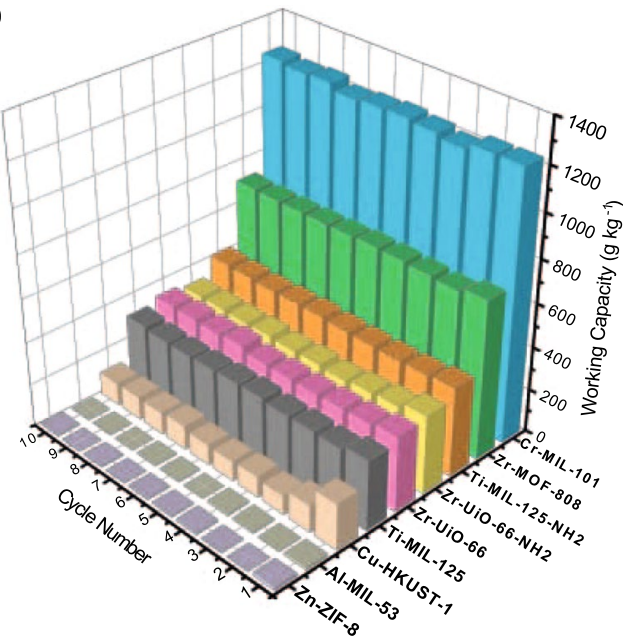

Figure 2. Water sorption properties of MOFs studied in present work. (a) Dynamic water vapor adsorption and desorption of Cr-MIL-101 in loose powder bed with a packing density of 0.65 at $1 \mathrm{~mm}$ thickness. Adsorption was performed at $70 \% \mathrm{RH}$ in solid spheres and $35^{\circ} \mathrm{C}$, desorption at $30 \% \mathrm{RH}$ and $60^{\circ} \mathrm{C}$ and shown in open spheres. (b) Working capacities of studied MOFs over ten consecutive sorption cycles. Adsorption cycles were performed at $70 \% \mathrm{RH}$ over $24 \mathrm{~h}$ at $22^{\circ} \mathrm{C}$; desorption cycles were performed at $60^{\circ} \mathrm{C}$ for $24 \mathrm{~h}$ under ambient humidity of ( 30\%). Zn-ZIF-8 (lavender), Al-MIL-53 (pickle), Cu-HKUST-1 (brown), Ti-MIL-125 (black), Zr-UiO-66 (pink), Ti-MIL-125- $\mathrm{NH}_{2}$ (orange), Cr-MIL-101 (blue), Zr-MOF-808 (green), and Zr-UiO-66-NH (yellow).

\begin{tabular}{|c|c|c|c|c|c|}
\hline MOF & $\mathrm{SA}^{a}\left(\mathbf{m}^{2} \mathrm{~g}^{-1}\right)$ & $\mathrm{V}_{\mathrm{p}}^{b}\left(\mathrm{~cm}^{3} \mathrm{~g}^{-1}\right)$ & Uptake capacity $^{c}\left(\mathrm{~g} \mathrm{~kg}^{-1}\right)$ & Working capacity ${ }^{d}\left(\mathrm{~g} \mathrm{~kg}^{-1}\right)$ & Water desorbed (\%) \\
\hline Ti-MIL-125 & 1153 & 0.47 & 323 & 313 & 97 \\
\hline Ti-MIL-125-NH & 1358 & 0.55 & 413 & 409 & 99 \\
\hline Zr-UiO-66 & 959 & 0.40 & 347 & 338 & 97 \\
\hline $\mathrm{Zr}-\mathrm{UiO}-66-\mathrm{NH}_{2}$ & 1109 & 0.46 & 364 & 355 & 98 \\
\hline Zr-MOF-808 & 1880 & 0.69 & 744 & 714 & 96 \\
\hline Cr-MIL-101 & 2579 & 1.63 & 1263 & 1246 & 98 \\
\hline Cu-HKUST-1 & 1512 & 0.41 & 218 & 105 & 48 \\
\hline Al-MIL-53 & 814 & 0.41 & 13 & 2 & 15 \\
\hline Zn-ZIF-8 & 1835 & 0.69 & 15 & 6 & 40 \\
\hline
\end{tabular}

Table 1. Summary MOFs studied with $\mathrm{N}_{2}$ gas for determining water vapor sorption properties. ${ }^{\text {aSurface area }}$ calculated from Brunauer-Emmett-Teller (BET) model analysis of $\mathrm{N}_{2}$ gas adsorption-desorption isotherm at $77 \mathrm{~K}$. ${ }^{b}$ Pore volume calculated at $p / p_{o}=0.4$ of $\mathrm{N}_{2}$ adsorption isotherm $(77 \mathrm{~K})$. ${ }^{c}$ Water vapor uptake capacity was determined gravimetrically; the value is taken from the first cycle adsorption at $70 \% \mathrm{RH}$ and $22^{\circ} \mathrm{C}$ at ambient pressure. ${ }^{\mathrm{d}}$ Working capacity was determined gravimetrically by the difference in the amount of water desorbed and adsorbed during water cycle stability and recovery studies.

the maximum harvested water is referred to as working capacity $\left(\mathrm{g}_{\mathrm{H} 2 \mathrm{O}} \mathrm{kg}^{-1} \mathrm{MOF}\right)$ and should be the same as the amount of water captured. This working capacity is related to the enthalpy of desorption, e.g., how much energy is needed for removing the physisorbed molecules from the MOF. Ideally, this desorption enthalpy should be high enough to allow water adsorption at low RH and remain bound until prescribed conditions. The desorption process should require little energy input and release all of the adsorbed water. To study this behavior, ten adsorption and desorption cycles were performed using the as-prepared MOFs, and the results are shown in Fig. 2. The adsorption cycle was performed on activated MOFs over $24 \mathrm{~h}$ at $70 \% \mathrm{RH}$ and $22^{\circ} \mathrm{C}$. In comparison, the desorption was performed at $30 \% \mathrm{RH}$ and $60^{\circ} \mathrm{C}$ to simulate conditions that can easily be achieved for a thermal-mediated desorption process with little to no energy input.

The gravimetric uptake capacity at equilibrium for water vapor has a strong correlation with total pore volume and is remarkably similar to previously reported data obtained using water vapor isotherm, with exception to Zn-ZIF-8 (Fig. 2, Table 1, and Supplementary Figs. S10-S18) ${ }^{20}$. Water recovery in Zn-ZIF-8 and Al-MIL-53 show recoveries of less than 6 and $2 \mathrm{gh}_{\mathrm{h} 2} \mathrm{~kg}^{-1} \mathrm{MOF}$ at the $10^{\text {th }}$ cycle, respectively. This performance comes from the hydrophilic nature and inability to uptake water vapor in both MOFs. Cu-HUKST-1 shows decreased working capacities over the first 3 cycles from 212 to $94 \mathrm{~g}_{\mathrm{H} 2 \mathrm{O}} \mathrm{kg}^{-1}$ MOF. This is significantly less than the $487 \mathrm{~g}_{\mathrm{H} 2 \mathrm{O}} \mathrm{kg}^{-1}$ MOF that the MOF adsorbed within the timeframe for the measurement. This finding shows that during the first desorption cycle, less than $50 \%$ of the initial adsorbed water can be removed under mild desorption conditions. This 


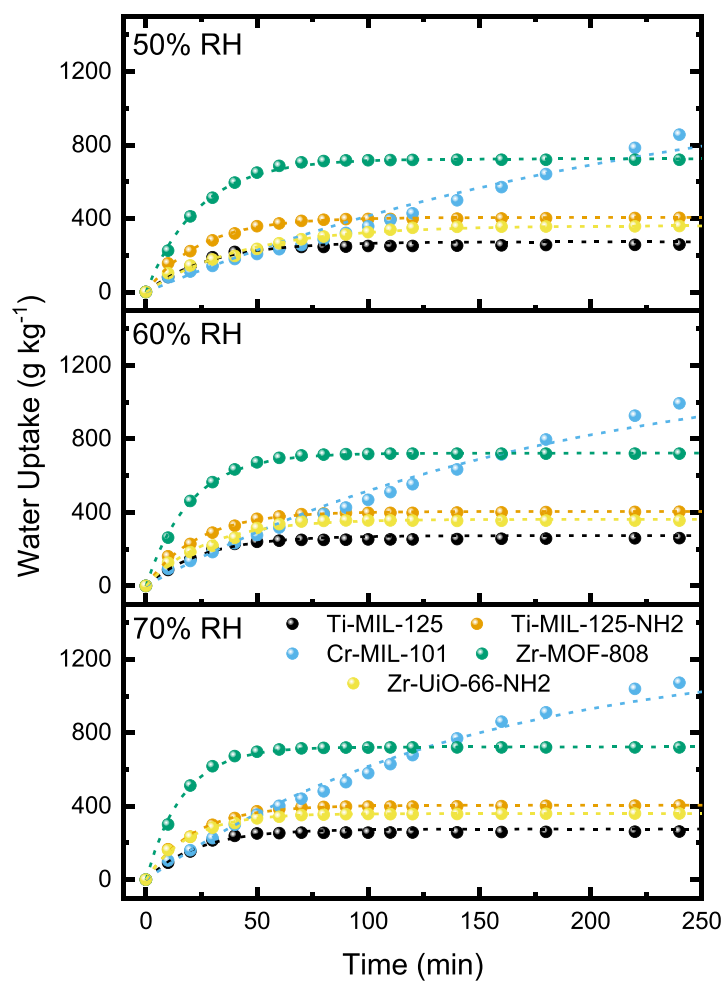

Figure 3. Water adsorption on loose powder bed samples with a packing porosity of $\sim 0.65-0.7$ and sample depths of $1 \mathrm{~mm}$ at $22^{\circ} \mathrm{C}$ and 50,60, and 70\% RH. MOF samples were fully activated prior to the adsorption kinetics study. Experimental results are shown as solid spheres, and LDF fitted plot as a dashed line in representative color. Ti-MIL-125 (black), Ti-MIL-125-NH (orange), Cr-MIL-101 (blue), Zr-MOF-808 (green), and $\mathrm{Zr}$-UiO-66- $\mathrm{NH}_{2}$ (yellow).

partial saturation after desorption is consistent with a strong interaction between water and the open $\mathrm{Cu}$ metal sites that cannot easily be removed under the desorption protocol of $60^{\circ} \mathrm{C}$ and $30 \% \mathrm{RH}$. Similar strong interactions have also been observed in silica gels ${ }^{21}$ and zeolites ${ }^{22}$ which require elevated temperatures, in some cases in excess of $300^{\circ} \mathrm{C}$, to remove water effectively. This amount of energy to regenerate a saturated material is not effective energetically or kinetically and can even cause decomposition.

The high-valence Ti-MIL-125 (246 $\left.\mathrm{g}_{\mathrm{H} 2 \mathrm{O}} \mathrm{kg}^{-1}{ }_{\mathrm{MOF}}\right)$ and Zr-UiO-66 (338 $\left.\mathrm{g}_{\mathrm{H} 2 \mathrm{O}} \mathrm{kg}^{-1}{ }_{\mathrm{MOF}}\right)$ show similar working capacities to their isoreticular counterparts, i.e., Ti-MIL-125- $\mathbf{N H}_{2}\left(393 \mathrm{~g}_{\mathrm{H} 2 \mathrm{O}} \mathrm{kg}^{-1} \mathrm{MOF}\right)$ and $\mathbf{Z r}-\mathbf{U i O}-\mathbf{6 6}-\mathbf{N H}_{2}$ $\left(320 \mathrm{~g}_{\mathrm{H} 2 \mathrm{O}} \mathrm{kg}^{-1}{ }_{\mathrm{MOF}}\right)$. The highest working capacity was observed in Zr-MOF-808 and Cr-MIL-101 of 616 and $1187 \mathrm{~g}_{\mathrm{H} 2 \mathrm{O}} \mathrm{kg}^{-1} \mathrm{MOF}$, respectively. Comprehensively, these working capacity results agree with the analogous pore volumes and BET surface areas in each respective MOF. Furthermore, the water vapor recovery remained unaltered throughout ten cycles. This outstanding resilience was confirmed with post-cycled powder x-ray diffraction (PXRD) analysis, which displays that there is no structural change in post-cycled MOFs (Supplementary Figs. S1-S9). There is no apparent trend in uptake capacities and surface functionalities.

Owing to their superior working capacities, Ti-MIL-125, Ti-MIL-125-NH, Cr-MIL-101, Zr-MOF-808, and $\mathrm{Zr}$-UiO-66- $\mathbf{N H}_{2}$ were further investigated using a carefully controlled parametric time study for understanding the water vapor adsorption-desorption processes. This can lead to further insight into the dynamic properties responsible for controlling water mass-transport and diffusivity coefficients. The diffusional behavior of water in particular materials is RH dependent and fluctuates with temperature making a comprehensive study difficult. Furthermore, the presence of heat transfer and more complex diffusional phenomenon often lead to spurious coefficients that represent a bulk average of the materials' intrinsic properties. For this purpose, we have limited our study to a thin-layer, loose grain powder bed reactor under high RHs to ensure reproducibility and relevance to practical applications. The reactor geometry, sorbent mass used, bed thickness, packing porosity, and crystallite sizes were all carefully controlled.

MOFs were compared by exposing them to various conditions over a $4000 \mathrm{~min}$ duration. The adsorption of water is recorded gravimetrically and plotted against time (Figs. $2 \mathrm{a}$ and 3 ). The water uptakes exhibited an apparent $1^{\text {st }}$ order behavior which can be modeled using linear driving force (LDF) and fitted accordingly with $\mathrm{R}^{2}>0.95$ in all accounts when modeled over the entire data range (Supplementary Figs. S38-S48, Table 2, and Supplementary Tables S1-S5 ${ }^{23}$. LDF offers analytical simplicity by stating the average sorbate uptake rate is determined by the products of the amount required to reach equilibrium and the rate constant. 


\begin{tabular}{|c|c|c|c|c|c|c|c|c|c|}
\hline \multirow[b]{2}{*}{ MOF } & \multicolumn{3}{|l|}{$50 \%$ RH } & \multicolumn{3}{|l|}{$60 \%$ RH } & \multicolumn{3}{|l|}{$70 \%$ RH } \\
\hline & $R_{0}\left(g_{k g}^{-1} \min ^{-1}\right)$ & $k\left(\min ^{-1}\right)$ & $D_{\mathrm{H} 2 \mathrm{O}}\left(\mathrm{cm}^{2} \min ^{-1}\right)$ & $\mathrm{R}_{0}\left(\mathrm{~g} \mathrm{~kg}^{-1} \min ^{-1}\right)$ & $k\left(\min ^{-1}\right)$ & $D_{\mathrm{H} 2 \mathrm{O}}\left(\mathrm{cm}^{2} \mathrm{~min}^{-1}\right)$ & $\mathrm{R}_{0}\left(\mathrm{~g} \mathrm{~kg}^{-1} \min ^{-1}\right)$ & $k\left(\min ^{-1}\right)$ & $D_{\mathrm{H} 2 \mathrm{O}}\left(\mathrm{cm}^{2} \mathrm{~min}^{-1}\right)$ \\
\hline Ti-MIL-125 & 7.15 & $2.50 \times 10^{-2}$ & $1.39 \times 10^{-4}$ & 7.86 & $2.74 \times 10^{-2}$ & $1.52 \times 10^{-4}$ & \begin{tabular}{|l|l|}
8.65 \\
\end{tabular} & $3.02 \times 10^{-2}$ & $1.68 \times 10^{-4}$ \\
\hline Ti-MIL-125-NH ${ }_{2}$ & $1.69 \times 10$ & $3.77 \times 10^{-2}$ & $5.82 \times 10^{-4}$ & $1.82 \times 10$ & $4.07 \times 10^{-2}$ & $6.29 \times 10^{-4}$ & $1.98 \times 10$ & $4.42 \times 10^{-2}$ & $6.84 \times 10^{-4}$ \\
\hline $\mathrm{Zr}-\mathrm{UiO}-66-\mathrm{NH}_{2}$ & 6.56 & $2.27 \times 10^{-2}$ & $2.58 \times 10^{-5}$ & $1.50 \times 10$ & $3.95 \times 10^{-2}$ & $5.90 \times 10^{-5}$ & $1.72 \times 10$ & $4.52 \times 10^{-2}$ & $6.67 \times 10^{-5}$ \\
\hline Zr-MOF-808 & $3.17 \times 10$ & $4.25 \times 10^{-2}$ & $1.80 \times 10^{-3}$ & $3.72 \times 10$ & $5.64 \times 10^{-2}$ & $2.12 \times 10^{-3}$ & $4.74 \times 10$ & $7.20 \times 10^{-2}$ & $2.70 \times 10^{-3}$ \\
\hline Cr-MIL-101 & 3.71 & $4.02 \times 10^{-3}$ & $4.42 \times 10^{-6}$ & 5.58 & $4.44 \times 10^{-3}$ & $6.65 \times 10^{-6}$ & 7.97 & $6.35 \times 10^{-3}$ & $9.50 \times 10^{-6}$ \\
\hline
\end{tabular}

Table 2. LDA fitting of experimental water vapor adsorptions results of variable RH on tested $\mathrm{MOFs}^{\mathrm{a}} .{ }^{\mathrm{a}}{ }^{\mathrm{L}}$ oose powder bed samples with a packing porosity of $\sim 0.65-0.7$ and sample depths of $1 \mathrm{~mm}$ at $22^{\circ} \mathrm{C}$. The average particle size was used to calculate the diffusional coefficient.

$$
\frac{d M_{t}}{d t}=k\left(M_{e}-M_{t}\right)
$$

where $M_{t}\left(\mathrm{~g}_{\mathrm{h} 2 \mathrm{o}} \mathrm{kg}^{-1}{ }_{\mathrm{MOF}}\right)$ is the amount of water vapor at time $t, \mathrm{M}_{e}\left(\mathrm{~g}_{\mathrm{h} 2 \mathrm{o}} \mathrm{kg}^{-1}{ }_{\mathrm{MOF}}\right)$ is the equilibrium uptake, and $k\left(\mathrm{~min}^{-1}\right)$ is the rate constant. This implies when plotting $\ln \left(\mathrm{M}_{e}-\mathrm{M}_{t}\right) \mathrm{M}_{e}^{-1}$ versus time $(t)$ the LDF mass-transfer coefficient can be obtained. It was described previously that the initial rate of the adsorption and desorption process, $\mathrm{R}_{0}$, is accurate at describing gravimetric water uptake at any given time, providing a numerical value to assess dynamic behavior ${ }^{4}$. Morphological and crystallite size information was determined using scanning electron microscopy (SEM) (Supplementary Figs. S27-S32) and dynamic light scattering (DLS) (Supplementary Figs. S33-S37), which was used to calculate water vapor intracrystalline diffusivity parameters based on the mass-transfer coefficients with the assumption that intercrystalline diffusivity being the rate-limiting step. $D_{H 2 O}$ can be calculated using the formula ${ }^{24}$ :

$$
D_{H 2 O}=\frac{k R_{M O F}^{2}}{15}
$$

where $D_{H 2 O}\left(\mathrm{~cm}^{2} \mathrm{~min}^{-1}\right)$ is the intercrystalline diffusivity coefficient, $R_{M O F}(\mathrm{~cm})$ is the average radius of MOF crystallites, and $k\left(\mathrm{~min}^{-1}\right)$ from Eq. 2 .

The dynamic water uptake was studied in triplicate for free bed samples with packing porosity of $\sim 0.65-0.7$ and bed depths of $1 \mathrm{~mm}$ at $22^{\circ} \mathrm{C}$ and $70 \%$ RH (Fig. 3 and Supplementary Fig. S38). Table 2 shows the results of LDF fitting and shows that the MOFs with polar functionalities uptake water significantly faster, exhibiting initial rates of 47.4, 19.8, and 17.2 $\mathrm{g} \mathrm{kg}^{-1} \mathrm{~min}^{-1}$ for MOF-808, $\mathbf{M I L}-\mathbf{1 2 5}-\mathbf{N H}_{2}$, and UiO-66- $\mathbf{N H}_{2}$, respectively. More nonpolar Ti-MIL-125 and Zr-MIL-101 show decreased uptake rates of 8.65 and $7.97 \mathrm{~g} \mathrm{~kg}^{-1} \mathrm{~min}^{-1}$, respectively. Impressively, all MOFs reached saturation in $60 \mathrm{~min}$, except for Cr-MIL-101, which took more than $4 \mathrm{~h}$. It is interesting to observe that while constraining the powder bed thickness to $1 \mathrm{~mm}$, the adsorption uptake dynamics are dominated by polar functionality and not the accessible surface area as Cr-MIL-101 has an uptake almost double of that of Zr-MOF-808 but exhibits an initial rate that is almost six times slower. As expected, when RH is incrementally decreased to $50 \%$, reduction in initial rates, adsorption kinetics, and diffusivities with minor deviations in saturation equilibriums was observed. Furthermore, the relative trends within each humidity trial between each MOF remain comparable.

We also found that ambient temperature played an important role on both adsorption and desorption, which in turn affected water vapor interactions and the physisorption process (Fig. 4, Table 3, and Supplementary Table S2). The investigation into the intracrystalline diffusivity barriers of water vapor adsorption process on MOFs activation energies was calculated at $70 \% \mathrm{RH}$ using the Arrhenius equation generated from data collected at 22,27 , and $35^{\circ} \mathrm{C}$. The results show that for more hydrophobic MOFs (Ti-MIL-125 and Cr-MIL-101), the uptake mass-transfer coefficient and initial rate are enhanced with increased temperature as expected. In contrast, more polar MOFs (Ti-MIL-125-NH, $\mathbf{Z r}-\mathrm{UiO}-66-\mathbf{N H}_{2}$, and $\mathbf{Z r - M O F - 8 0 8 ) ~ e x p e r i e n c e d ~ a ~ d e c r e a s e ~ i n ~ t h e i r ~ r a t e ~}$ constants at elevated temperatures. The latter can be attributed to two key reasons: (1) the enhanced mass-transfer rates from the diffusion of water vapor prompted by elevated temperature; (2) the attractive interactions responsible for the physisorption of water adsorption is exothermic and effectively weakened as temperature increases and facilitates desorption ${ }^{25}$. Consequently, adsorption kinetics are negatively affected by the increase in temperature. This becomes more evident in MOFs that have strong interactions with water vapor. In short, MOFs with polar functional groups and high enthalpy of adsorption, e.g., more hydrophilic MOFs, tend to have a more negative dependence on temperature than those with a lower enthalpy of adsorption regardless of pore size, volume, and accessible surface area (Ti-MIL-125 and Cr-MIL-101). Interestingly, Ti-MIL-125- $\mathbf{N H}_{2}$ and Zr-UiO-66-NH have similar available surface areas, water uptakes, metal-ions, and linker properties: but have a significant difference in the activation energies of -8.17 and $-31.7 \mathrm{~kJ} \mathrm{~mol}^{-1}$, respectively. This variance in hydrophilicity can also be observed in the influx point of 24 and 16\% RH (Supplementary Table S1) respectively for Ti-MIL-125- $\mathbf{N H}_{2}$ and $\mathbf{Z r - U i O}-66-\mathbf{N H}_{2}$, exhibiting that the latter is more hydrophilic in nature. This temperature dependence is an important finding that cannot be determined from water vapor adsorption isotherms alone and emphasizes the importance of time-dependent uptake studies to help in materials design. There was no appreciable impact from temperatures on the equilibrium reached, excluding for Zr-MOF-808, decreasing from 742 to $560 \mathrm{~g} \mathrm{~kg}^{-1}$ for 22 and $35^{\circ} \mathrm{C}$, respectively. 
a

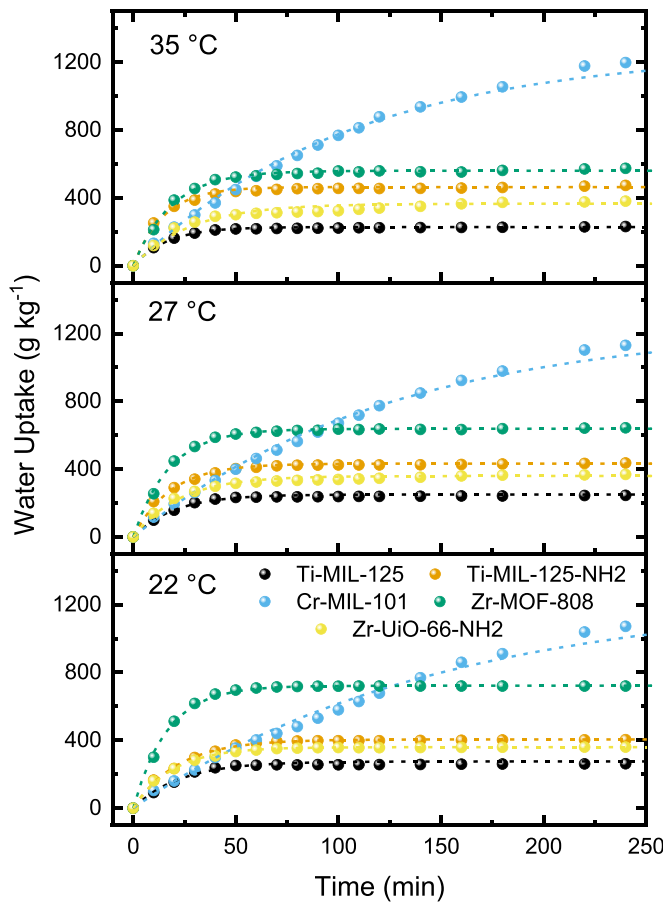

b

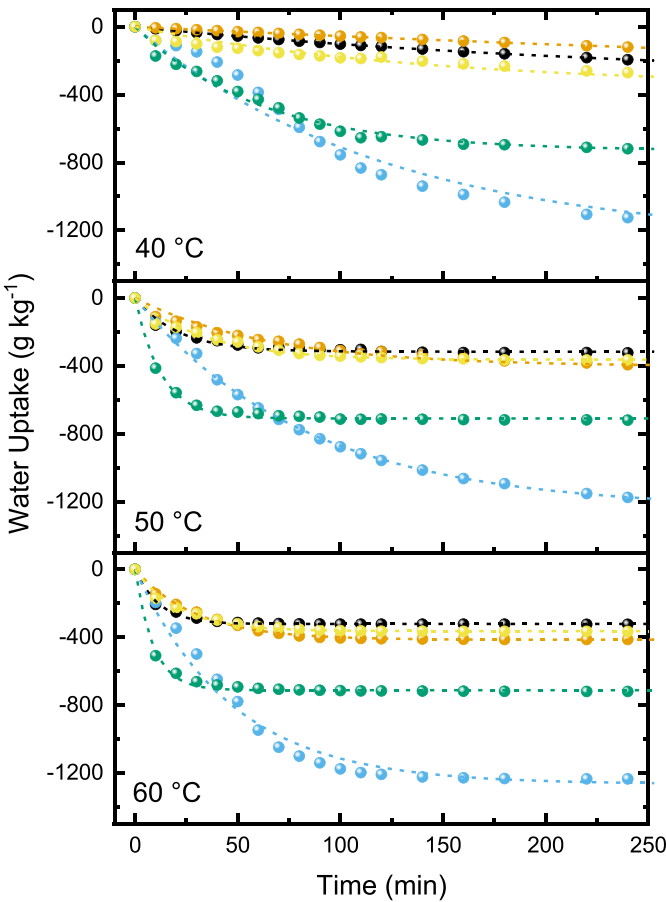

Figure 4. Thermal dependence of water vapor dynamics on fixed powder bed samples with packing porosities of $\sim 0.65-0.7$ and sample depth of $1 \mathrm{~mm}$. (a) Water vapor adsorption at $70 \% \mathrm{RH}$ and 22,27 , and $35^{\circ} \mathrm{C}$ and (b) water vapor desorption at $30 \% \mathrm{RH}$ and 40,50 , and $60^{\circ} \mathrm{C}$. Experimental results are shown as solid spheres, and LDF fitted plot as a dashed line in representative color. Ti-MIL-125 (black), Ti-MIL-125-NH $\mathbf{N H}_{2}$ (orange), CrMIL-101 (blue), Zr-MOF-808 (green), and Zr-UiO-66- $\mathrm{NH}_{2}$ (yellow).

\begin{tabular}{|c|c|c|c|c|}
\hline \multirow[b]{2}{*}{ MOF } & \multicolumn{2}{|c|}{$R_{0}\left(\mathrm{~g} \mathrm{~kg}^{-1} \min ^{-1}\right)$} & \multirow[b]{2}{*}{$\mathrm{E}_{\mathrm{a}}^{b}\left(\mathrm{~kJ} \mathrm{~mol}^{-1}\right)$} & \multirow[b]{2}{*}{$\mathbf{R}_{\text {adj }}^{2}$} \\
\hline & $22^{\circ} \mathrm{C}$ & $35^{\circ} \mathrm{C}$ & & \\
\hline Ti-MIL-125 & 8.65 & $1.02 \times 10$ & 9.62 & 0.999 \\
\hline Ti-MIL-125- $\mathrm{NH}_{2}$ & $1.98 \times 10$ & $1.72 \times 10$ & -8.17 & 0.988 \\
\hline $\mathrm{Zr}-\mathrm{UiO}-66-\mathrm{NH}_{2}$ & $1.72 \times 10$ & 9.82 & -31.7 & 0.949 \\
\hline Zr-MOF-808 & $4.74 \times 10$ & $2.60 \times 10$ & -34.1 & 0.966 \\
\hline Cr-MIL-101 & 7.97 & 9.87 & 12.3 & 0.978 \\
\hline
\end{tabular}

Table 3. The rate dependence and activation energies of water uptake at $70 \% \mathrm{RH}^{\mathrm{a}}$. ${ }^{\mathrm{a}}$ Loose powder bed samples

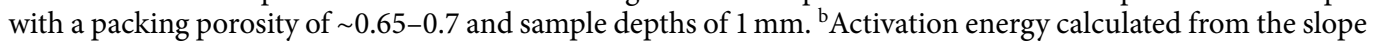
from the Arrhenius plot of the kinetic rate constants vs. temperatures.

The subsequent desorption was performed after samples were equilibrated at $70 \% \mathrm{RH}$ and $22^{\circ} \mathrm{C}$ over 24 hours (Fig. 4b and Supplementary Figs. S46-S48). Studies were completed at 30\% RH and temperatures of 40, 50, and $60^{\circ} \mathrm{C}$. There is no apparent trend in the desorption rates, with respect to porosity or functionality, of all MOF that show initial desorption rates ranging from $0.632-10.8 \mathrm{~g} \mathrm{~kg}^{-1} \mathrm{~min}^{-1}$ at $40^{\circ} \mathrm{C}$ to $16.7-74.5 \mathrm{~g} \mathrm{~kg}^{-1} \mathrm{~min}^{-1}$ at $60^{\circ} \mathrm{C}$. Zr-MOF-808 exhibits the most attractive dynamic desorption properties under every condition studied.

The maximum potential water production can be estimated from the combination of the dynamic adsorption and desorption data (Table 4). It should be noted that the presented data assumes that collection efficiency is $100 \%$ and that the relative humidity changes with the adsorption and desorption cycle. The numbers represent an ideal number that is not representative of what is possible, but it provides an upper limit to what is potentially achievable with intelligent heating and chilling to modulate dew point, water desorption from saturated sorbent, and water condensation. Furthermore, this module, when used in conjunction with a fan to facilitate water vapor diffusion, will be energy-intensive and requires appropriate balancing with available energy sources that could be farmed with photovoltaics or other means. Surprisingly, the nonpolar Ti-MIL-125 has the potential to harvest up to $3.96 \mathrm{~L}_{\mathrm{H} 2 \mathrm{O}} \mathrm{kg}^{-1}{ }_{\mathrm{MOF}}$ day $^{-1}$, almost half that of the acidic-functionalized $\mathrm{Zr}$-MOF-808 (8.66) and similar to the amino-functionalized Ti-MIL-125- $\mathbf{N H}_{2}$ (4.17) and $\mathbf{Z r}-\mathbf{U i O}-66-\mathbf{N H}_{2}$ (3.97). Cr-MIL-101 can generate up to $1.67 \mathrm{~L}_{\mathrm{H} 2 \mathrm{O}} \mathrm{kg}^{-1}$ MOF $\mathrm{day}^{-1}$, only being able to be cycled 1.3 times per day. This is significantly smaller than that of Ti-MIL-125, which is noteworthy, given it has over four times the working capacity and shows a maximum daily water output of less than half. It is important to point out that the proper selection of materials is dependent upon 


\begin{tabular}{|c|c|c|c|}
\hline MOF & Working Capacity $\left(\mathrm{g} \mathrm{kg}^{-1}\right)$ & Cycle time $^{b}(\mathrm{~min})$ & Recovery potential $\left(\mathrm{L} \mathrm{kg}^{-1}\right.$ day $\left.^{-1}\right)$ \\
\hline Ti-MIL-125 & 275 & 100 & 3.96 \\
\hline Ti-MIL-125- $\mathrm{NH}_{2}$ & 405 & 140 & 4.17 \\
\hline $\mathrm{Zr}-\mathrm{UiO}-66-\mathrm{NH}_{2}$ & 359 & 130 & 3.97 \\
\hline Zr-MOF-808 & 722 & 120 & 8.66 \\
\hline Cr-MIL-101 & 1252 & 1080 & 1.67 \\
\hline
\end{tabular}

Table 4. Water recovery potential of MOFs evaluated ${ }^{\mathrm{a}}$. ${ }^{\mathrm{W}}$ Water adsorption at $22^{\circ} \mathrm{C}, 70 \% \mathrm{RH}$, packing porosities of $\sim 0.65-0.7$, and sample depth of $1 \mathrm{~mm}$. Water desorption at $60^{\circ} \mathrm{C}, 30 \% \mathrm{RH}$, packing porosities of $\sim 0.65-0.7$, and sample depth of $1 \mathrm{~mm}$. ${ }^{\mathrm{b}} \mathrm{Cycle}$ time calculated from the sum of the time it takes for complete saturation during adsorption and the time for complete desorption for respective MOF.

environmental constraints such as the RH and ability to harvest energy. If there is little available energy, there is a restriction on the number of cycles that can be facilitated by additional peripherals to aid in heating, cooling, and circulating air.

Loose-grain powder bed reactors are simple but can suffer from limited diffusivity as bed thickness increases. The role of intracrystalline diffusivity is an import factor in understanding water uptake. For practical considerations, there needs to be a balance between total additional energy, sorption kinetics, and volumetric constraints on the specific system to be employed. The inter- and intra-crystalline mass transport, and by extension intracrystalline diffusivity, strongly influence the adsorption-desorption properties (disregarding thermal parameters) in loose-grain powder beds. Furthermore, if there is limited environmental energy that can be harvested to power thermal swings and circulation fans, the number of cycles needs to be optimized accordingly. The diffusional time scale $(t)$ and intercrystalline diffusion $\left(\mathrm{D}_{\text {intrer }}\right)$ are greatly affected by sample bed thickness $\left(\mathrm{H}_{\mathrm{MOF}}\right)$, as $t \sim \mathrm{H}_{\mathrm{MOF}}{ }^{2}$ $\mathrm{D}_{\text {intrer }}{ }^{-17}$. Adsorption dynamics collected at $70 \% \mathrm{RH}$ and $22^{\circ} \mathrm{C}$ for the studied MOFs with packing porosities of $\sim 0.65-0.7$ and a sample depth of $2 \mathrm{~mm}$ are presented in Fig. $5 \mathrm{a}$ and Table 5 . Sample depths of $1 \mathrm{~mm}$ are primarily controlled from the intrinsic properties of individual crystallites; however, as sample depths are increased to $2 \mathrm{~mm}$, and presumably beyond, all studied MOFs have similar initial rates of uptake. This regime is no longer dominated by specific morphology and functionality; uptake dynamics are determined by intracrystalline diffusivity, with intercrystalline diffusivities being negligible. The most critical material property to consider is the adsorption capacity at equilibrium. Consequently, Cr-MIL-101 was also studied at bed depths of 5 and $10 \mathrm{~mm}$ (Fig. 5b). The $1^{\text {st }}$ order LDA can no longer adequately model the uptake kinetics for Cr-MIL-101 at these depths and a pseudo-zero models appears to be a better model. This region is no longer kinetically favorable, needing over $24 \mathrm{~h}$ to reach equilibrium in both scenarios. Design of a powder bed system that operates under similar conditions as used in this study would require stacked shelves with laminar flow to optimize volumetric uptake capacity such has been recently demonstrated ${ }^{4}$. Regardless, these findings illustrate the significance of powder bed thickness on materials selection, which is primarily driven by the chemical functionality and structural topology. We would like to point out that sample thickness will be affected by thermodynamic factors, e.g., heat generation and dissipation, that can both affect the sorbent material's performance. As sample thickness increases, the intracrystalline effects will become a more dominant influence that limits the adsorption and desorption kinetics. We see that under our testing conditions there is limited dependence on the specific properties of the MOFs as the depth is increased.

\section{Conclusion}

We investigated a series of MOFs with a wide variety of structural topologies and chemical functionalities for atmospheric water harvesting and studied their structure-property relationships. This work presents the first quantitative direct comparative investigation of MOFs evaluating different simulated operational conditions to facilitate proper materials selection to optimize materials and system design. Interestingly, thermal studies revealed that hydrophilic MOFs have a negative dependence with increasing temperature whilst nonpolar MOFs show enhanced uptake over the same temperature regime. Modulation of powder bed depth suggests the competition between intracrystalline and intercrystalline diffusivities control water vapor dynamics in different thickness regimes, revealing that all MOFs studied have similar uptake dynamics at a bed thickness of $2 \mathrm{~mm}$, regardless of MOF properties. At a powder bed thickness of $1 \mathrm{~mm}$, the role that surface functionality and topology have on water harvesting is significant, showing the performance of Zr-MOF-808 is superior in most scenarios and, under ideal conditions, could generate up to $8.66 \mathrm{~L}_{\mathrm{H} 2 \mathrm{O}} \mathrm{kg}^{-1}{ }_{\mathrm{MOF}}$ day ${ }^{-1}$. Unfortunately, Zr-MOF-808 has an influx point at $36 \% \mathrm{RH}$, which limits its applications in arid environments with relative humidities typically ranging from $10-30 \%$. The results presented here enhance our understanding of MOFs as solid-state sorbent materials and of the role that the structure-property relationship plays in dictating materials selection. In our future work, we plan to investigate additional MOFs with similar properties to Zr-MOF-808, e.g., those with low RH influx points, high surface areas, and polar functional properties for investigation towards arid conditions. Furthermore, studying MOFs in different geometries such as pellets or extruded in monolithic honeycomb structures, from a volumetric constraint, optimizing thermodynamic properties, as a composite, or even as host matrixes for deliquescent materials, may allow for enhanced uptake performance while maintaining a fixed shape and size. All these are interesting and worth investigating in future work. These will be reported in future reports. 

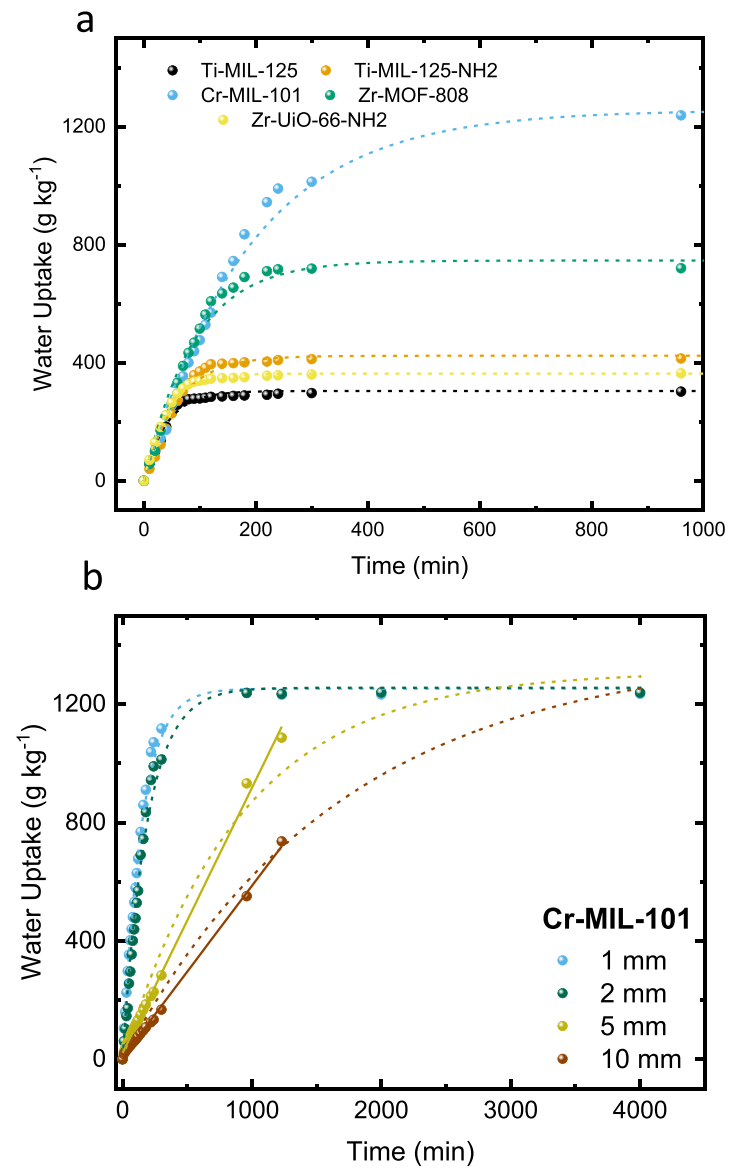

Figure 5. Exposed surface-area-to-volume ratio dependence of water vapor dynamics on fixed powder bed samples at $70 \% \mathrm{RH}$ and $22^{\circ} \mathrm{C}$. (a) Water vapor adsorption of studied MOFs with a packing porosity of

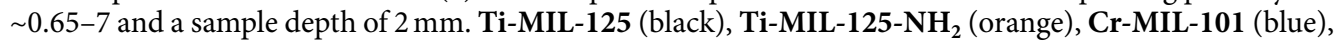
Zr-MOF-808 (green), and Zr-UiO-66- $\mathbf{N H}_{2}$ (yellow). (b) Water vapor adsorption of Cr-MIL-101 at a packing porosity of $\sim 0.65-7$ and sample depth of 1 (blue), 2(green), 5 (yellow), and 10 (red) mm. Experimental results are shown as solid spheres, LDF fitted plot as a dashed line, and pseudo-zero order fitted as a solid line in representative color.

\begin{tabular}{|l|l|l|}
\hline \multirow{2}{*}{ MOF } & \multicolumn{2}{|l|}{$\mathbf{R}_{\mathbf{0}}\left(\mathrm{g} \mathrm{kg}^{-\mathbf{1}} \mathbf{m i n}^{-\mathbf{1}}\right)$} \\
\cline { 2 - 3 } & depth $=\mathbf{1} \mathbf{~ m m}$ & depth $=\mathbf{2 ~} \mathbf{m m}$ \\
\hline Ti-MIL-125 & 8.65 & 7.11 \\
\hline Ti-MIL-125-NH & & 7.58 \\
\hline Zr-UiO-66-NH & $1.98 \times 10$ & 9.68 \\
\hline Zr-MOF-808 & $1.72 \times 10$ & 7.45 \\
\hline Cr-MIL-101 & $4.74 \times 10$ & 6.69 \\
\hline
\end{tabular}

Table 5. Rate dependence of water uptake on powder bed depth at $70 \% \mathrm{RH}$ and $22^{\circ} \mathrm{C}$.

\section{Methods}

Materials. Chromium (III) nitrate nonahydrate $\left(\mathrm{Cr}\left(\mathrm{NO}_{3}\right)_{3} \bullet 9 \mathrm{H}_{2} \mathrm{O}, \geq 99 \%\right)$, iron(III) nitrate nonahydrate $\left(\mathrm{Fe}\left(\mathrm{NO}_{3}\right)_{3} \bullet 9 \mathrm{H}_{2} \mathrm{O}, \geq 98 \%\right)$, titanium (IV) isopropoxide $\left(\mathrm{Ti}\left(\mathrm{O}{ }^{i} \mathrm{Pr}\right)_{4}, 97 \%\right)$, zirconium(IV) chloride $\left(\mathrm{ZrCl}_{4}, \geq 99.5 \%\right)$, aluminum nitrate nonahydrate $\left(\mathrm{Al}\left(\mathrm{NO}_{3}\right)_{3} \bullet 9 \mathrm{H}_{2} \mathrm{O}, \geq 98 \%\right)$, zinc nitrate hexahydrate $\left(\mathrm{Zn}\left(\mathrm{NO}_{3}\right)_{2} \bullet 6 \mathrm{H} 2 \mathrm{O}, 98 \%\right)$ were purchased from Sigma-Aldrich. The organic linker moieties: 1,4-benzenedicarboxylic acid $\left(\mathrm{H}_{2} \mathrm{BDC}, 98 \%\right)$, 2-amino-1,4-benzenedicarboxylic acid $\left(\mathrm{H}_{2} \mathrm{BDC}-\mathrm{NH}_{2}, 99 \%\right), 1,3,5$-benzenetricarboxylic acid $\left(\mathrm{H}_{3} \mathrm{BTC}\right.$, $\left.95 \%\right)$, and 2-methylimidazole (HMIM, 99\%) were obtained from Sigma-Aldrich. Acid modulation was performed using benzoic acid (HBC, 99.5\%) and nitric acid $\left(\mathrm{HNO}_{3}, 70 \%\right)$. Ultra-high purity $\mathrm{He}$ and $\mathrm{N}_{2}$ gas (99.999\%) were purchased from Airgas. Anhydrous solvents ( $\mathrm{MeOH}$ and $\mathrm{DMF}$ ) were purchased from Sigma-Aldrich in Sureseal bottles. Unless specified otherwise, all reactions were performed under ambient laboratory conditions, and all reagents were used as received with no further purification from a commercial source. 
Materials characterization. Powder X-ray diffraction (PXRD) was performed under ambient conditions on a PANalytical Empyrean Series 2 diffractometer in a Bragg-Brentano geometry using a Ni-filtered lined-focused $\mathrm{CuK}_{\alpha}$ radiation $(\lambda=1.54 \AA$ ). Data was collected from 5-40 2 $\theta$-degrees. Simulation of PXRD powder pattern was calculated using Visualization for Electronic and Structural Analysis (VESTA) v. 3.4.4 from the CIF file of the respective MOF retrieved from the respective reference.

Nitrogen gas adsorption isotherms were performed on a Quantachrome NOVA 2200e surface area and porosity analyzer at $77 \mathrm{~K}$. Prior to the tests, all samples were thermally activated overnight at $100^{\circ} \mathrm{C}$ under $0.01 \mathrm{mmHg}$. Helium gas was used to measure the dead space volume prior to measurements. Brunauer-Emmett-Teller (BET) surface areas were determined by linear least-square fitting of the BET plot, the upper working limits were provided by the Rouquerol analysis. Following the procedure by Snurr et al., the volume of the monolayer, BET surface areas, and C-constant were determined for the $\mathrm{MOFs}^{26}$.

Thermogravimetric analysis (TGA) was performed using a TA Instruments Q5000 from room temperature to $600^{\circ} \mathrm{C}$ at $10^{\circ} \mathrm{C} / \mathrm{min}$ under a continuous nitrogen flow. Test results were analyzed using Universal Analysis Software.

To get insights into the compatibility of different phases, the characteristics of the rubber fracture surfaces were observed via scanning electron microscopy (SEM) using a Thermo Scientific ${ }^{\mathrm{TM}}$ Scios $^{\mathrm{TM}}$ DualBeam $^{\mathrm{TM}}$ operated at $5 \mathrm{kV}$. All samples were first fractured in a consistent manner after soaking in liquid nitrogen for 5 minutes. Fractured sample surfaces were sputter-coated with iridium prior to SEM imaging.

The particle size of MOF was measured using a Dynamic Light Scattering (DLS) analyzer- Malvern Zetasizer Nano ZS. A sample of $0.100 \mathrm{mg}$ was dispersed in methanol and sonicated for $1 \mathrm{~h}$ prior to analysis.

Preparation of MOFs. MOFs were prepared according to previously reported methods: Ti-MIL-125 ${ }^{13}$,

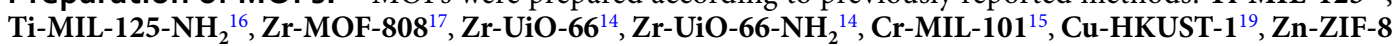
$(\mathbf{Z n})^{18}$, and Al-MIL-53 ${ }^{12}$. Isolation was performed by vacuum filtration after MOF samples cooled to room temperature naturally. Samples were washed with DMF $(3 \times, \sim 25 \mathrm{~mL})$ followed by $\mathrm{CHCl}_{3}(3 \times, \sim 25 \mathrm{~mL})$. Samples were then generally immersed in $25 \mathrm{~mL}$ of $\mathrm{MeOH}$ for $2 \mathrm{~d}$ in a desiccator, replacing solvent through this time $(6 \times)$ ). Residual $\mathrm{MeOH}$ was decanted off and finally dried under dynamic vacuum $(0.01 \mathrm{mmHg})$ at $120^{\circ} \mathrm{C}$ for $16 \mathrm{~h}$ to yield activated samples and stored under Ar atmosphere until further studies. Characterization results are consistent with the corresponding literature data, confirming proper synthesis and activation procedure.

Water uptake studies. Gravimetric uptake studies were performed in a home-built environmental chamber using a TaoTronics ultrasonic cool mist humidifier charged with Ultrapure water (Milli-Q system) and using joule heating as the heat source for relative humidity $(\mathrm{RH})$ control. A loose grain powder bed was used to simulate working conditions. Fully activated MOF powder was placed flat inside polypropylene beakers with a flat bottom and parallel sidewalls. The temperature and RH were respectively controlled at $295 \mathrm{~K}$ and $70 \%$, unless specified otherwise, at ambient pressure. The RH and temperatures of the system were verified using a high-accuracy secondary thermocouple and humidity sensor that shows variations of less than $5 \% \mathrm{RH}$ and $2{ }^{\circ} \mathrm{C}$. A calibrated balance was used to record masses at predetermined time intervals.

Measurements were first performed for several days to assess ideal experimental conditions. A loose grain powder bed geometry with a fixed packing porosity $(\sim 0.65-0.7)$ and thickness was changed from $1 \mathrm{~mm}$ to $10 \mathrm{~mm}$. The porosity of the MOF powder bed $(\varphi)$ was calculated from the following equation:

$$
\varphi=1-\frac{\rho_{\text {AppMOF }}}{\rho_{\text {PowderPart }}}
$$

where $\rho_{A p D M O F}$ is the apparent density of the MOF and $\rho_{\text {PowderPart }}$ is powder particle density which is determined from pycnometry and BET determined pore volumes. Kim et al. determined, in the case of Zr-MOF-801, that a porosity above 0.5 as necessary to ensure molecular and Knudsen diffusion behavior is avoided ${ }^{7}$. We expect similar results in the case of MOFs investigated here, for they have similar crystallite sizes.

Prior to water desorption, MOFs were allowed to saturate with water at $70 \% \mathrm{RH}$ and $22^{\circ} \mathrm{C}$ for at least $24 \mathrm{~h}$ to minimize measurement uncertainties, which could potentially arise from not being at equilibrium. A Lindberg Blue- $\mathrm{M}$ vacuum oven operated at ambient pressure and $30 \% \mathrm{RH}$ to simulate environmental conditions would be set at the target temperature. Samples were placed into the oven and weigh periodically. The $\mathrm{RH}$ and temperatures of the system were verified using a high-accuracy secondary thermocouple and humidity sensor that shows variations of less than $6 \% \mathrm{RH}$ and $2^{\circ} \mathrm{C}$.

Water cycle stability and recovery, the working capacity $\left(\mathrm{g}_{\mathrm{h} 2 \mathrm{o}} \mathrm{kg}^{-1} \mathrm{MOF}_{\mathrm{F}}\right)$, was performed in triplicate over 10 cycles on a $100 \mathrm{mg}$ scale. Adsorption cycles were performed at $70 \% \mathrm{RH}$ over $24 \mathrm{~h}$. Desorption cycles were performed at $60^{\circ} \mathrm{C}$ for $24 \mathrm{~h}$ under ambient $\mathrm{RH}(\sim 30 \%)$. Working capacity in the context of this study is defined as the amount of water vapor that is desorbed.

Kinetic measurements were performed through the course of $4000 \mathrm{~min}$. To minimize Knudsen diffusion and potential thermal effects, measurements were first taken of thickness and packing porosity of $1 \mathrm{~mm}$ and 0.65 , respectively. The adsorption kinetics for $70 \% \mathrm{RH}$ and $22^{\circ} \mathrm{C}$ was performed in triplicate to ensure reproducibility (Fig. S38) - all remaining kinetics plots as a single measurement. The effect of RH was studied by maintaining the temperature at $22^{\circ} \mathrm{C}$ and fluctuation in the $\mathrm{RH}$ from $50-70 \% \mathrm{RH}$. Thermal effects of adsorption were studied by maintaining $\mathrm{RH}$ at $70 \%$ and varying the temperature from 22 to 27 and $35^{\circ} \mathrm{C}$. The effect of thickness was studied at $1,2,5$, and $10 \mathrm{~mm}$. Desorption studies were performed at 60,50 , and $40^{\circ} \mathrm{C}$.

\section{Data availability}

All data generated or analyzed during this study are included in this published article (and its Supplementary Information file). 
Received: 27 October 2019; Accepted: 14 January 2020;

Published online: 30 January 2020

\section{References}

1. Cosgrove, W. Water Futures: the evolution of water scenarios. Current Opinion in Environmental Sustainability 5, 559-565 (2013).

2. Elimelech, M. \& Phillip, W. A. The future of seawater desalination: energy, technology, and the environment. Science 333, 712-717 (2011).

3. Al-Karaghouli, A. \& Kazmerski, L. L. Energy consumption and water production cost of conventional and renewable-energypowered desalination processes. Renewable and Sustainable Energy Reviews 24, 343-356 (2013).

4. Hanikel, N. et al. Rapid Cycling and Exceptional Yield in a Metal-Organic Framework Water Harvester. ACS Central Science, https:// doi.org/10.1021/acscentsci.9b00745 (2019).

5. Burtch, N. C., Jasuja, H. \& Walton, K. S. Water stability and adsorption in metal-organic frameworks. Chemical Reviews 114, 10575-10612 (2014).

6. Stock, N. \& Biswas, S. Synthesis of metal-organic frameworks (MOFs): routes to various MOF topologies, morphologies, and composites. Chemical Reviews 112, 933-969 (2011).

7. Kim, H. et al. Water harvesting from air with metal-organic frameworks powered by natural sunlight. Science 356, 430-434 (2017).

8. Ribeiro, A. M. et al. Adsorption equilibrium and kinetics of water vapor on different adsorbents. Industrial \& Engineering Chemistry Research 47, 7019-7026 (2008).

9. Wade, C. R., Corrales-Sanchez, T., Narayan, T. C. \& Dincă, M. Postsynthetic tuning of hydrophilicity in pyrazolate MOFs to modulate water adsorption properties. Energy \& Environmental Science 6, 2172-2177 (2013).

10. Akiyama, G. et al. Effect of functional groups in MIL-101 on water sorption behavior. Microporous and Mesoporous Materials 157, 89-93 (2012)

11. Canivet, J. et al. Structure-property relationships of water adsorption in metal-organic frameworks. New Journal of Chemistry 38, 3102-3111 (2014).

12. Ahnfeldt, T. et al. Synthesis and modification of a functionalized 3D open-framework structure with MIL-53 topology. Inorganic Chemistry 48, 3057-3064 (2009).

13. McNamara, N. D., Neumann, G. T., Masko, E. T., Urban, J. A. \& Hicks, J. C. Catalytic performance and stability of (V) MIL-47 and (Ti) MIL-125 in the oxidative desulfurization of heterocyclic aromatic sulfur compounds. Journal of Catalysis 305, 217-226 (2013).

14. Katz, M. J. et al. A facile synthesis of UiO-66, UiO-67 and their derivatives. Chemical Communications 49, 9449-9451 (2013).

15. Zhao, T. et al. High-yield, fluoride-free and large-scale synthesis of MIL-101 (Cr). Dalton Transactions 44, 16791-16801 (2015).

16. Logan, M. W. et al. Systematic variation of the optical bandgap in titanium based isoreticular metal-organic frameworks for photocatalytic reduction of CO 2 under blue light. Journal of Materials Chemistry A 5, 11854-11863 (2017).

17. Li, Z.-Q., Yang, J.-C., Sui, K.-W. \& Yin, N. Facile synthesis of metal-organic framework MOF-808 for arsenic removal. Materials Letters 160, 412-414 (2015).

18. Cravillon, J. et al. Rapid room-temperature synthesis and characterization of nanocrystals of a prototypical zeolitic imidazolate framework. Chemistry of Materials 21, 1410-1412 (2009).

19. Tranchemontagne, D. J., Hunt, J. R. \& Yaghi, O. M. Room temperature synthesis of metal-organic frameworks: MOF-5, MOF-74, MOF-177, MOF-199, and IRMOF-0. Tetrahedron 64, 8553-8557 (2008).

20. Abtab, S. M. T. et al. Reticular chemistry in action: A hydrolytically stable MOF capturing twice its weight in adsorbed water. Chem 4, 94-105 (2018).

21. Li, X., Li, Z., Xia, Q. \& Xi, H. Effects of pore sizes of porous silica gels on desorption activation energy of water vapour. Applied Thermal Engineering 27, 869-876 (2007).

22. Simo, M., Sivashanmugam, S., Brown, C. J. \& Hlavacek, V. Adsorption/desorption of water and ethanol on 3A zeolite in nearadiabatic fixed bed. Industrial \& Engineering Chemistry Research 48, 9247-9260 (2009).

23. Sircar, S. \& Hufton, J. Why does the linear driving force model for adsorption kinetics work? Adsorption 6, 137-147 (2000).

24. Rolando, M. \& Roque-Maherbe, R. Adsorption and diffusion in nanoporous materials. (CRC Press, Taylor and Francis Group, Boca Raton, FL, 2007).

25. Saha, P. \& Chowdhury, S. Insight into Adsorption Thermodynamics. (InTech, Vienna, 2011).

26. Walton, K. S. \& Snurr, R. Q. Applicability of the BET method for determining surface areas of microporous metal-organic frameworks. Journal of the American Chemical Society 129, 8552-8556 (2007).

\section{Acknowledgements}

This research was supported by the Independent Research and Development (IRAD) Fund from the Research and Exploratory Development Mission Area of the Johns Hopkins University Applied Physics Laboratory. The authors would like to thank Dr. Tim Montabano, Mr. John King, Mr. Daniel Rose, and Ms. Hadas Elazar-Mittelman for some experimental assistance.

\section{Author contributions}

M.L. contributed to the M.O.F. synthesis and adsorption study. S.L. contributed to the materials characterization and testing, and Z.X. contributed to the overall concept design of this work.

\section{Competing interests}

The authors declare no competing interests.

\section{Additional information}

Supplementary information is available for this paper at https://doi.org/10.1038/s41598-020-58405-9.

Correspondence and requests for materials should be addressed to Z.X.

Reprints and permissions information is available at www.nature.com/reprints.

Publisher's note Springer Nature remains neutral with regard to jurisdictional claims in published maps and institutional affiliations. 
(c) (i) Open Access This article is licensed under a Creative Commons Attribution 4.0 International License, which permits use, sharing, adaptation, distribution and reproduction in any medium or format, as long as you give appropriate credit to the original author(s) and the source, provide a link to the Creative Commons license, and indicate if changes were made. The images or other third party material in this article are included in the article's Creative Commons license, unless indicated otherwise in a credit line to the material. If material is not included in the article's Creative Commons license and your intended use is not permitted by statutory regulation or exceeds the permitted use, you will need to obtain permission directly from the copyright holder. To view a copy of this license, visit http://creativecommons.org/licenses/by/4.0/.

(C) The Author(s) 2020 